Taste

\section{Flavour of the week in}

\section{signalling pathways}

Cell 112, 293-301 (2003)

Tastes come in five flavours: salty, sour, bitter, sweet or umami - the taste of amino acids. Taste buds in the mouth contain many taste-receptor cells, which relay flavours, via nerves, to taste centres in the brain. According to one theory, a single taste-receptor cell carries receptors for the different flavours, each using a different intracellular signalling pathway to convey the sensation to nerves. But Yifeng Zhang et al. find that sweet, bitter and umami are detected by distinct cells, using different receptors but the same signalling pathway.

The sweet, bitter and umami receptors known are mainly found on separate tastereceptor cells. But all these cells contain the enzyme phospholipase $\mathrm{C} \beta 2$ and the ion channel TRPM5. Zhang et al. show that mice lacking either phospholipase $C \beta 2$ or TRPM5 cannot taste sweet, bitter or umami, yet have normal salt and sour perception. The authors propose that phospholipase C 32 and TRPM5 are part of the same signalling pathway, in which the former activates the latter. Restoring phospholipase $\mathrm{C} \beta 2$ only in cells carrying bitter receptors restores the response to bitter tastes, but not to sweetness or umami, confirming that individual taste-receptor cells detect only one of these three flavours. Marie-Thérèse Heemels

\section{Atomic imaging}

\section{Like peas in a 'nanopod'}

Phys. Rev. Lett. 90, 055506 (2003)

The cage-like structure of carbon fullerenes can encapsulate other elements, and the properties of these complexes depend strongly on the precise location of the guest atoms. This is particularly true of the dimetallofullerenes - complexes that contain two metal atoms within each fullerene molecule.

Using high-resolution transmission electron microscopy (HRTEM), K. Suenaga and colleagues have determined the location of metal atoms in individual molecules of scandium dimetallofullerene, $\mathrm{Sc}_{2} @ \mathrm{C}_{84}$. The scandium atoms undergo both covalent and ionic interactions with their $\mathrm{C}_{84}$ cage, and three different isomers of this molecule have been predicted. But one isomer, with a peculiar nonpolar arrangement of scandium atoms, had never been observed.

The use of HRTEM to directly identify specific atomic species, particularly such a light element as scandium, is challenging. Suenaga et al. filled a single-walled carbon nanotube with $\mathrm{Sc}_{2} @ \mathrm{C}_{84}$ molecules purified

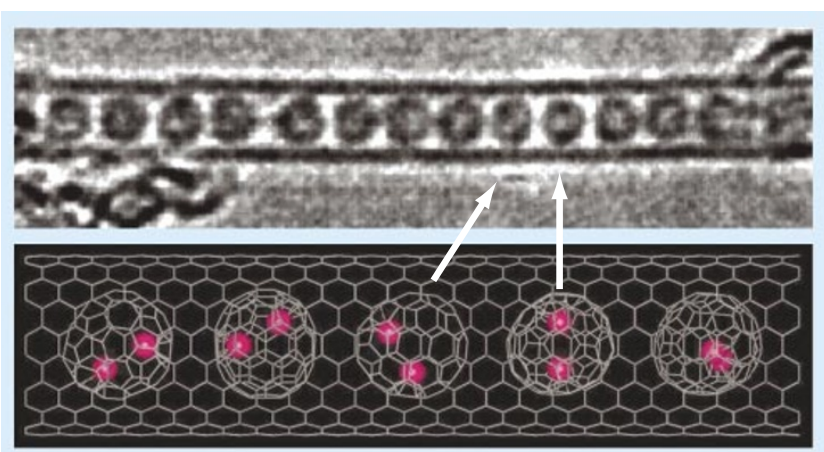

In this atomicresolution image (top) of dimetallofullerene molecules inside a carbon nanotube, the positions of the scandium atoms encapsulated in the fullerene can be seen to match the theoretical prediction (bottom).

to contain only the isomer of interest. By comparing their HRTEM images of these fullerene 'peapods' with simulations (see picture), the authors identified the positions of the scandium atoms in the fullerene isomer, and confirm that they match the theoretical prediction.

Ed Gerstner

\section{Animal behaviour}

\section{Heredity and the bolder bird}

Proc. R. Soc. Lond. B doi:10.1098/rspb.2002.2300 (2003)

Bold great tits have adventurous children, whereas the offspring of timid birds stay closer to home. The discovery of a link between birds' personality and their dispersal behaviour points to this trait as having a powerful effect on the genetic structure of populations.

Niels J. Dingemanse and his colleagues captured wild great tits (Parus major) and put them in a novel environment - a sealed room containing artificial trees. Some birds were quick to explore their surroundings, others more hesitant. After the birds' return to the real world, the researchers found that the offspring of the bolder individuals dispersed the farthest, whereas those of timid tits stayed close to the parental nest. They also found that birds that had migrated into the study population were more adventurous than locally born individuals, and that bold females - the sex that, in birds, tends to leave home dispersed farther after fledging than shy ones.

About half of the variation in a great tit's personality can be explained by heredity. So the geographical distribution of genes for boldness will affect the spread of other genes, and also the population dynamics of the species - what birds colonize new environments, for example. John Whitfield

\section{Biophysics}

\section{Unzipping your genes}

Proc. Natl Acad. Sci. USA 100, 1694-1699 (2003)

The unzipping of DNA in the cell for replication typically proceeds in stops and starts. Generally, this seems to be caused by faults or twists, which prompt pauses for
DNA repair. But experiments by Claudia Danilowicz et al. show that even flawless DNA unzips sporadically. The researchers tethered one strand of viral DNA to a glass capillary, and pulled, with constant force, on a microscopic magnetic bead attached to the other strand. The separation of the strands occurred in a series of jumps, repeatedly becoming stuck for several minutes or so.

This behaviour is expected, because G-C base pairs are slightly more strongly bound together than A-T pairs. So G-C-rich sequences will be harder to prise apart. Because unbinding is thermally activated, the duration of the stalled spells is randomly determined. If the separation force is small enough, the unzipping process may pause for hours on end.

Philip Ball

\section{Neurobiology}

\section{Firing patterns add up}

Neuron 37, 499-511 (2003)

When nerve cells signal from the eye to the brain, they do so in harmony, say Mark J. Schnitzer and Markus Meister. They have built a new algorithm that can detect the cells talking in unison.

Groups of neurons are thought to carry coded information about the world by firing in coordinated sequences. But computers struggle to detect these patterns from the vast number of possible combinations hidden in the thousands of signals recorded from neurons.

To get round this, Schnitzer and Meister built an iterative algorithm. Rather than looking for a firing pattern among all signals and all cells, it first looks for a match between pairs of cells - and then searches for others that fire in sync. Using their program, the researchers found sets of up to seven cells that signal together in the salamander's retina. These account for more than half of all the electrical 'spikes' they recorded. The authors suggest that each firing pattern, rather than each neuron, carries a separate message to the brain. This could communicate better visual detail about an image, because there are many more possible patterns than individual neurons.

Helen Pearson 